\section{Sopping up SAP}

\section{By Tim Fulmer, Senior Writer}

A team of European researchers has developed an antibody-small molecule combination that treats amyloidosis by lowering levels of a protein called serum amyloid $\mathrm{P}$ component in both circulation and solid organs. ${ }^{1}$ The therapy has shown efficacy in mouse models of the disease, and GlaxoSmithKline plc has exclusively in-licensed the IP. The pharma is preparing a humanized antibody against the protein for clinical studies.

Amyloidosis is caused by the misfolding of

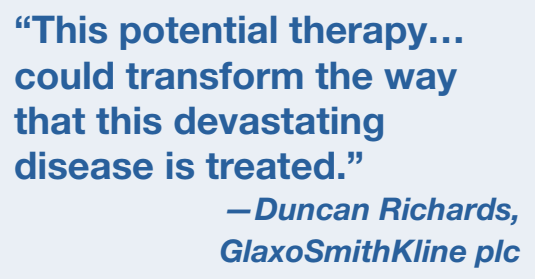

only or no treatment. Additional mechanistic studies showed that macrophages play a key role in the amyloid clearance.

Pentraxin Therapeutics Ltd., an IP holding company founded by Pepys and spun out of University College London, holds rights to the college's patent applications covering the combination therapy to treat or prevent amyloidosis. Last year, Pentraxin granted GSK exclusive, worldwide rights to co-develop and commercialize the CPHPC/anti-SAP antibody combination in return for an undisclosed up-front payment plus milestones and royalties.

What initially attracted GSK to the combination therapy was "its ability to clear existing amyloid deposits. That clearly differentiates this potential therapy from standard of care and could transform the way that this devastating disease is treated," said Duncan Richards, clinical director of the GSK Academic Discovery Performance Unit and project leader on the Pentraxin-GSK collaboration.

GSK "is now actively preparing an anti-SAP mAb for future clinical studies," said Richards, proteins that normally are soluble but instead get deposited as insoluble amyloid fibrils in one or more organs. This deposition leads to organ failure and, in some cases, death.

Misfolding of at least 20 different proteins can lead to a variety of amyloidosis disorders that affect organs throughout the body. There are no approved therapies to clear established fibrils and deposits. Instead, standard of care involves a variety of anti-inflammatory and cytotoxic compounds that prevent the formation of new deposits by lowering levels of one of the misfolded proteins that cause deposits. ${ }^{2}$

In addition to the presence of misfolded protein, amyloid fibrils contain other proteins that can promote the formation and persistence of amyloid deposits. One of those fibril-promoting proteins is serum amyloid P component (SAP; APCS), which has been shown to protect amyloid fibrils from degradation in vitro and contribute to their formation in vivo through an unclear mechanism.,

Thus, a team led by Mark Pepys, director of the Centre for Amyloidosis \& Acute Phase Proteins and professor of medicine at the University College London Medical School, hypothesized that depleting SAP in serum and amyloid deposits could prevent new deposit formation and clear out established ones.

In 2002, the team designed a small molecule called CPHPC ( $R$-1-[6-[R-2-carboxy-pyrrolidin-1-yl]-6-oxo-hexanoyl] pyrrolidine2 -carboxylic acid) that bound soluble SAP and cleared it from circulation, preventing the accumulation of SAP in the organs of both amyloidosis animal models and about 50 amyloidosis patients enrolled in a small pilot study.

However, CPHPC was unable to clear SAP in established amyloid deposits. For that task, the researchers decided to use antibodies that recognize and bind multiple epitopes of SAP in both its soluble and fibrilbound forms.

Now, new findings published in Nature show that an anti-SAP antibody plus CPHPC can lower amyloid deposits in the liver and spleen of transgenic mice with established amyloidosis compared with CPHPC who declined to disclose further details. He acknowledged that a combination therapy "will complicate the clinical and regulatory process, but given the substantial potential here we feel the effort is justified."

\section{Beyond SAP}

Pepys told SciBX that he and his colleagues at University College London are working very closely with GSK to carry out "additional animal studies to further characterize the mechanism of action of the dual therapy and inform dosing and treatment schedules for the first human studies. We're also developing new MRI methods to monitor the effect of the therapy on amyloid deposits in vivo."

In addition to the group's positive results with SAP, Pepys' team has tackled another soluble protein - transthyretin (TTR) - that causes transthyretin amyloidosis. The hereditary disorder is caused by single point mutations in TTR that lead to deposits of the misfolded plasma protein.

Based on the effectiveness of CPHPC in targeting a soluble protein like SAP, Pepys' team hypothesized that a similar approach could work with mutant TTR.

Thus, the researchers designed two bivalent small molecules, designated mds84 and 4ajm15, which bound mutant TTR with high affinity. The two compounds had the added and unexpected bonus of stabilizing wild-type TTR.

Those in vitro findings, which were published in the Proceedings of the National Academy of Sciences, ${ }^{7}$ are covered by patent applications from Pentraxin that have been exclusively licensed to an undisclosed pharma, Pepys told SciBX.

He said that next steps would include "a developability screen of the lead compound to determine whether or not any additional modification and optimization will be required to progress it as a therapeutic candidate."

Fulmer, T. SciBX 3(45); doi:10.1038/scibx.2010.1341

Published online Nov. 18, 2010 


\section{REFERENCES}

1. Bodin, K. et al. Nature; published online Oct. 20, 2010; doi:10.1038/nature09494

Contact: Mark B. Pepys, University College London, London, U.K. e-mail: m.pepys@ucl.ac.uk

2. Gillmore, J.D. \& Hawkins, P.N. Nat. Clin. Pract. Nephrol. 2 , 263-270 (2006)

3. Tennent, G.A. et al. Proc. Natl. Acad. Sci. USA 92, 4299-4303 (1995)

4. Botto, M. et al. Nat. Med. 3, 855-859 (1997)

5. Gillmore, J.D. et al. Br. J. Haematol. 148, 760-767 (2010)
6. Pepys, M.B. et al. Nature 417, 254-259 (2002)

7. Kolstoe, S.E. et al. Proc. Natl. Acad. Sci. USA; published online Nov. 11, 2010; doi:10.1073/pnas.1008255107

Contact: Mark B. Pepys, University College London, London, U.K. e-mail:m.pepys@ucl.ac.uk

COMPANIES AND INSTITUTIONS MENTIONED

GlaxoSmithKline plc (LSE:GSK; NYSE:GSK), London, U.K.

Pentraxin Therapeutics Ltd., London, U.K.

University College London Medical School, London, U.K. 